\title{
Contact lens-related Pseudomonas aeruginosa keratitis in a 49-year-old woman
}

\author{
Harald Gjerde MD, Anuradha Mishra MD
}

Cite as: CMAJ 2018 January 15;190:E54. doi: 10.1503/cmaj.171165

A 49-year-old woman with well-controlled diabetes presented with progressive pain, redness and visual decline in her left eye after taking out her daily disposable contact lenses three days previously. On visual acuity testing in this eye, she was able to perceive hand motions. Slitlamp examination showed a large corneal ulcer and hypopyon (Figure 1A). Corneal cultures were taken and the patient was treated for presumed infectious keratitis. Broad-spectrum antibiotic coverage was started with hourly ofloxacin and fortified tobramycin eye drops, along with ciprofloxacin ointment and ciprofloxacin taken orally. The cultures grew Pseudomonas aeruginosa. Once the defect had epithelialized, we added a topical steroid. When the infection had cleared, a large corneal scar remained (Figure 1B) and her visual acuity remained limited to being able to count fingers. Secondary to the prolonged inflammation, the patient developed high intraocular pressure in her left eye, which could not be controlled with medical management and required surgical intervention. A corneal transplant is planned to optimize her visual potential.

Common causative agents of infectious keratitis are $P$. aeruginosa, Staphylococcus aureus, coagulase-negative Staphylococci and Streptococcus pneumoniae. ${ }^{1,2}$ The virulent $P$. aeruginosa is most commonly associated with contact lens use. ${ }^{1}$ Pseudomonas ulcers are more severe at presentation and difficult to treat, leading to worse visual outcomes. ${ }^{1,2}$ We encourage physicians to strongly consider infectious keratitis in the differential for an acute red eye, particularly in a contact lens wearer. Risk factors for contact lens-related infectious keratitis include overuse of contact lenses, overnight wear, diabetes, trauma, poor hand and lens hygiene, and blepharitis. ${ }^{3,4}$ Because of its rapid progression, sometimes within hours, infectious keratitis should be considered a medical emergency, requiring an urgent ophthalmology consult. Contact lenses should be discontinued during treatment. Topical broad-spectrum antibiotics (such as fluoroquinolones, often combined with fortified aminoglycoside or vancomycin) every 30-60 minutes should be started promptly. Steroid eye drops may be considered, but should be begun and monitored by an ophthalmologist. ${ }^{2}$

\section{References}

1. Green M, Apel A, Stapleton F. Risk factors and causative organisms in microbial keratitis. Cornea 2008;27:22-7.

2. Lakhundi S, Siddiqui R, Khan NA. Pathogenesis of microbial keratitis. Microb Pathog 2017;104:97-109.

3. Stapleton F, Naduvilath T, Keay L, et al. Risk factors and causative organisms in microbial keratitis in daily disposable contact lens wear. PLoS One 2017;12: e0181343.

4. Weissman BA, Mondino BJ. Risk factors for contact lens associated microbial keratitis. Cont Lens Anterior Eye 2002;25:3-9.

\section{Competing interests: None declared.}

This article has been peer reviewed.

The authors have obtained patient consent.

Affiliation: Department of Ophthalmology and Visual Sciences, Dalhousie University, Halifax, NS

Correspondence to: Harald Gjerde, harald.gjerde@dal.ca 